\title{
Electrical conductivity of the hippocampal CA1 layers and application to current-source-density analysis
}

\author{
J. Holsheimer \\ Biomedical Engineering Division, Department of Electrical Engineering, University of Twente, P. O. Box 217, \\ NL-7500 AE Enschede, The Netherlands
}

Summary. The microstructure of the layers in the hippocampal CA1 area suggests that differences may exist between the electrical conductivities of these layers. In order to quantify these differences a sinusoidal current was applied to hippocampal slices in a bathing medium and potential differences were measured between pairs of neighbouring electrodes from an array. The maximum relative conductivity $(100 \%)$ was found in the middle part of str. radiatum, with a gradual decrease towards the fissure $(84 \%)$. There was also a gradual decrease towards the alveus $(70 \%)$, but in str. pyramidale the relative conductivity was only $42 \%$. No differences were observed between the laminar conductivities of normal hippocampal slices and slices generating spontaneous interictal bursts. These results were used to carry out a one-dimensional CSD analysis of field potentials evoked by Schaffer collateral stimulation. Despite the differences in conductivity, the homogeneous and the inhomogeneous CSD approximations did not lead to differences in the spatial distribution of sources and sinks and only gave some differences in the current density, especially at the pyramidal layer and its close environment.

Key words: Hippocampus - CA1 area - Laminar conductivity - Current source density - In vitro hippocampal slice

\section{Introduction}

For a precise interpretation of field potentials in the brain it is important to consider the fact that inhomogeneities and anisotropy may be present in the conducting medium. Such inhomogeneities and anisotropy in electrical conductivity should be taken into account when the transmembrane current density is estimated from the field potential distribution. This method is known as the current-sourcedensity (CSD) analysis (Freeman and Stone 1969; Nicholson and Freeman 1975). It has been applied to several parts of the brain, like areas of the cerebral and cerebellar cortex and the hippocampus (Haberly and Shepherd 1973; Kwan and Murphy 1974; Mitzdorf and Singer 1978; Leung 1979; Rappelsberger et al. 1981). For a recent review see Mitzdorf (1985). Conductivities have been measured in several cortical areas (Haberly and Shepherd 1973; Hoeltzell and Dykes 1979; Rappelsberger et al. 1981), but not in the hippocampus.

The electrical conductivity is related to the ratio of extra- and intra-cellular space and the tortuosity of the interstitial space (Gardner-Medwin 1980). In the hippocampus the (large) cell somata are densely packed in the pyramidal layer, while in the dendritic layers there is a dense network of fine dendritic arborizations and axons. Therefore we may expect that the extracellular conductivity of the pyramidal layer is different from the surrounding dendritic layers.

For the interpretation of epileptiform phenomena in the CA1 area of the in-vitro hippocampal slice preparation we used spatial field potential analysis methods. The CA1 area has a relatively simple laminar organization, with the dendritic trees of the pyramidal cells arranged in parallel, and the activity of these cells is highly synchronized during evoked potentials (Andersen et al. 1971) and epileptiform activity (Schwartzkroin and Prince 1978). Therefore a simplified one-dimensional version of CSD analysis - perpendicular to the laminated structure - could be used and electrical conductivities had to be determined only in this direction. Because we only needed the relative values of current sources and sinks, it was sufficient to know the relative conductivities of the CA1 layers. The laminar con- 


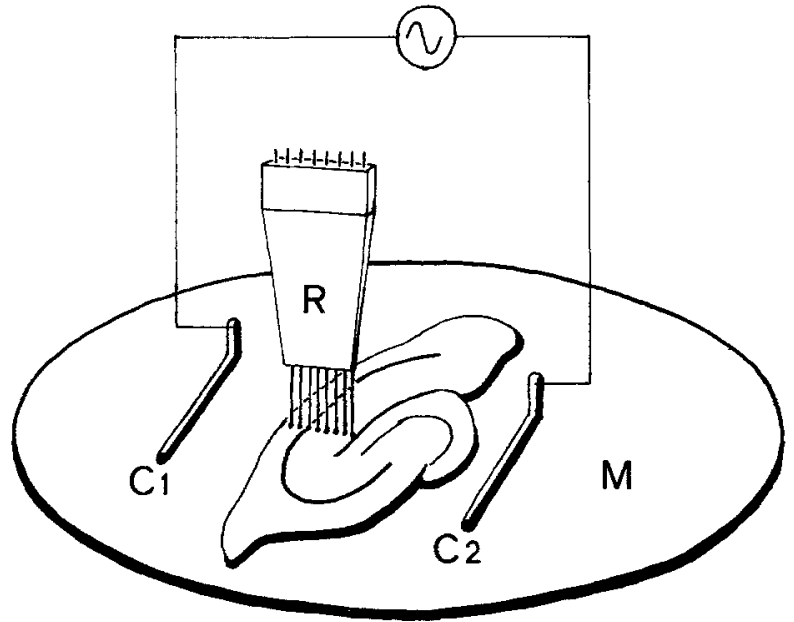

Fig. 1. Experimental set-up. Nylon gauze (M) with hippocampal slice at the surface of the perfusion chamber; recording electrode array $(\mathrm{R})$ in the hippocampal CA1 field, perpendicular to the laminated structure; two current electrodes (C1-2) perpendicular to the electrode array in the bathing medium surrounding the slice

ductivities were measured in normal slices and in those generating epileptiform burst activity.

\section{Methods}

In-vitro transverse hippocampal slices ( $0.6 \mathrm{~mm}$ thick) of 6 guinea pigs $(400-600 \mathrm{~g})$ were used. Conductivity measurements were made in a perfusion chamber at a temperature of approx. $32.5^{\circ} \mathrm{C}$. The composition (in $\mathrm{mM}$ ) of the perfusate was: $\mathrm{NaCl} \mathrm{124,} \mathrm{KCl} 5$, $\mathrm{NaH}_{2} \mathrm{PO}_{4} 1.24, \mathrm{MgSO}_{4} 1.3, \mathrm{NaHCO}_{3} 26, \mathrm{CaCl}_{2} 2$ and glucose 10 . Humidified gas $\left(95 \% \mathrm{O}_{2}\right.$ and $\left.5 \% \mathrm{CO}_{2}\right)$ was blown over the slices, lying on a nylon gauze just underneath the surface of the bathing medium in the perfusion chamber.

An isolated current source was used in combination with two wire electrodes (stainless steel, $2.5 \mathrm{~mm}$ long, diameter $0.1 \mathrm{~mm}$ ) in parallel at a distance of $3.5 \mathrm{~mm}$. These current electrodes were placed in the perfusion chamber on both sides of the slice, in parallel with the layers in CA1 where the measurements were made (Fig. 1). In order to investigate the frequency-dependence of the tissue conductivity $(\sigma)$, currents were delivered at frequencies of $100 \mathrm{~Hz}$ and $1 \mathrm{kHz}$.

Potentials were recorded with the eight-electrode array developed in our laboratory (Verloop and Holsheimer 1984), in combination with high-impedance preamplifiers $\left(10^{15} \Omega / / 0.6 \mathrm{pF}\right)$. The reference electrode was mounted at the bottom of the perfusion chamber. The array, with electrode diameters of $32 \mu \mathrm{m}$ and intervals of $0.1 \mathrm{~mm}$, was placed in CA1 perpendicular to the laminae and the current electrodes. With respect to the current electrodes the electrode array was situated in the middle part, where the current density (I) was almost constant (Fig. 1). In this configuration the potential difference $(\mathrm{dV})$ between a pair of neighbouring electrodes is linearly related to the tissue impedance (R) between this electrode pair; $d V=R . I=I / \sigma$, with $I=$ constant. In order to compensate for (small) inhomogeneities in I and for small differences in the spacings between the recording electrodes, control measurements were made in the bathing medium after removal of the slice, with the current electrodes and recording electrodes in the same positions as before.
In order to avoid effects of the injected sinusoidal current on the electrical activity of the neurons, which could affect the conductivity of the tissue, a current density was chosen which gave a $\mathrm{dV}$ between two subsequent recording electrodes of $0.7 \mathrm{mV}$ (peak-peak) or less. At these values we did not observe any difference from the situation without injected current: no action potentials were recorded from the pyramidal layer. In order to suppress recording noise, the amplified signals were fed into matched narrow-band filters $(100 \mathrm{~Hz}$ or $1 \mathrm{kHz})$. The filtered signals from seven pairs of electrodes were subsequently fed into a differential amplifier and the output was measured by a digital voltmeter. The seven values obtained from a slice were then divided by the corresponding control values obtained from recording in the bathing medium. The mean of the seven corrected values was calculated and the data obtained were expressed as a percentage of this mean. These values were considered as the relative tissue impedances $\left(R_{\text {rel }}\right)$ between the corresponding electrode pairs $\left(R_{\mathrm{rel}}=\mathrm{dV} / \mathrm{I}\right.$, with $\mathrm{I}=$ constant $)$.

\section{Results}

Frequency dependence of the laminar conductivity

Conductivity measurements were carried out on several slices with sinusoidal currents at $100 \mathrm{~Hz}$ and $1 \mathrm{kHz}$. No difference was observed at these frequencies, neither in the distribution of $\mathrm{dV}$ between the electrode pairs, nor in the mean value of $\mathrm{dV}$ from all pairs. Therefore it was concluded that the volume conductor of the CA1 field is resistive, at least in the frequency range used, which corresponds to the hippocampal field potentials.

\section{Effect of injected current density on the laminar conductivity}

In some measurements the current density was varied, in order to see whether an effect on the tissue conductivity could be observed. The average value of $\mathrm{dV}$ was varied between $0.3-0.8 \mathrm{mV}$ (peak-peak) at both $100 \mathrm{~Hz}$ and $1 \mathrm{kHz}$. No changes of $\mathrm{dV}$ were observed in this range of current densities. Therefore an effect of the injected sinusoidal current on the laminar conductivity can be excluded in our experiments.

\section{Laminar distribution of conductivity}

The relative tissue impedance $R_{\text {rel }}$ of the layers in the CA1 field was measured in 10 slices with a sinusoidal current of $1 \mathrm{kHz}$. In each slice several measurements were made at depths varying from $0.05-0.20 \mathrm{~mm}$ from the surface. In each series the current electrodes and the recording electrode array were kept in the same position as regards the hippocampal CA1 

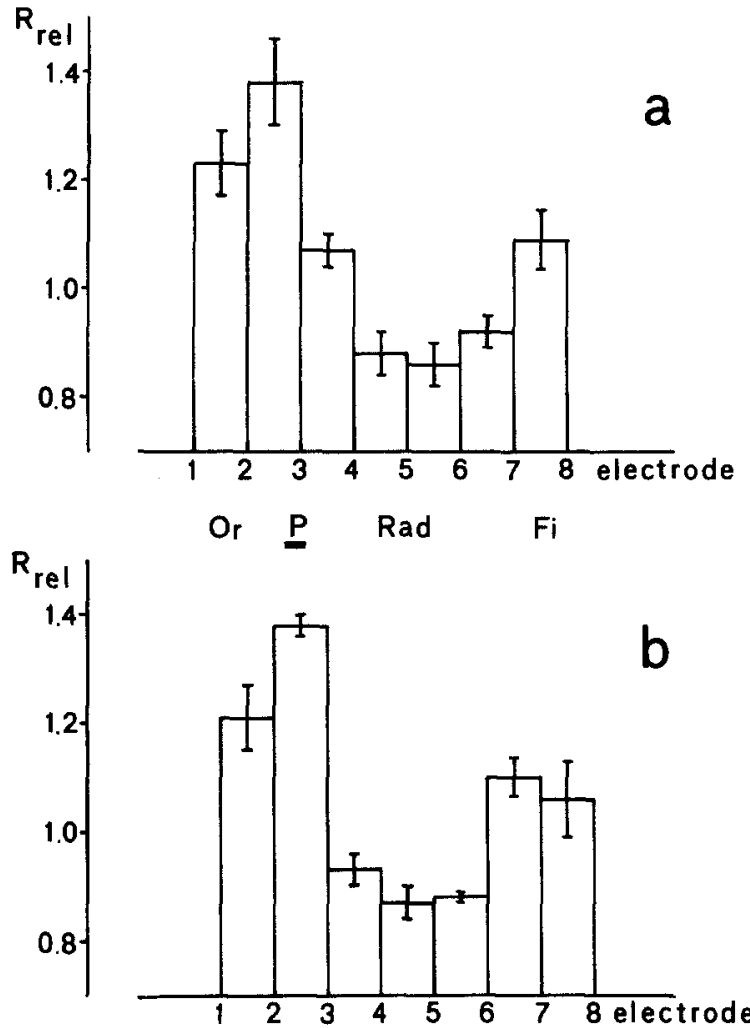

Fig. 2. a Mean and standard deviation of relative tissue resistance $\mathrm{R}_{\mathrm{rel}}$ between seven electrode pairs in the CA1 field measured at several depths in one slice at a current frequency of $1 \mathrm{kHz}$. b The same data from the electrode array, moved towards the fissure (Fi) by half the electrode interval; Or: stratum oriens; P: stratum pyramidale, Rad: stratum radiatum

field. Because the small pyramidal layer shows up very well in the slice as a dark line, the positioning of the recording electrode array was referred to this layer. In a series of slices the third electrode of the array was placed in the pyramidal layer, as when recording epileptiform activity. The first electrode was then at about the border of the alveus and str. oriens, and the hippocampal fissure was at the level of electrode 8 or between 7 and 8 , depending on the local width of str. radiatum and str. lacunosummoleculare. When placing the electrode array deeper in the slice, only a slight decrease of the mean $\mathrm{dV}$ was observed, with no change in the ratio of $\mathrm{dV}$ from the electrode pairs. The mean and standard deviation of each $R_{\text {rel }}$ were calculated from 6-8 measurements at varying depths in each slice. The results obtained from one slice are shown in Fig. 2a. The least variation and a small gradient of $R_{\text {rel }}$ were found in the middle part of str. radiatum. In order to compare the results from different slices, the distribution of $\mathrm{R}_{\mathrm{rel}}$ was normalized at a value of 0.88 in this part of stratum radiatum.

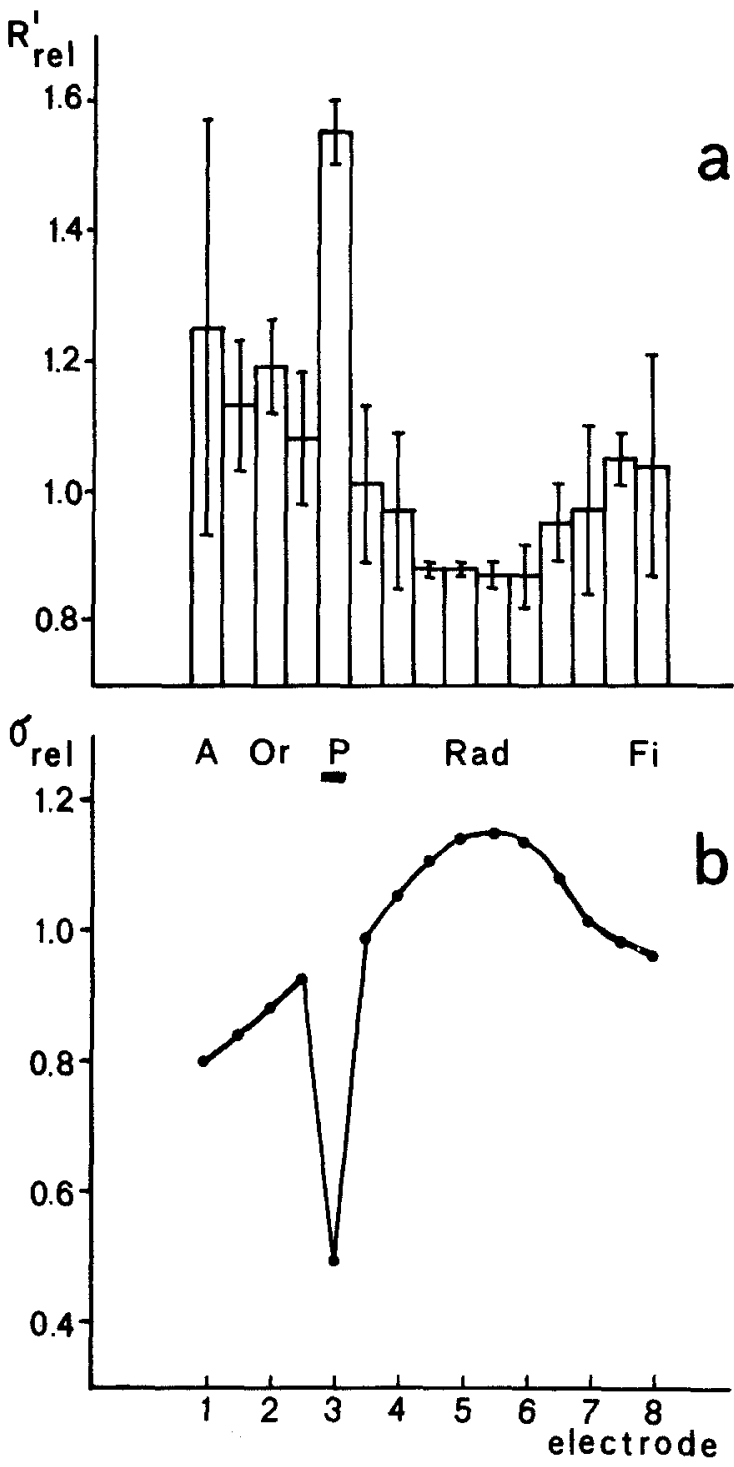

Fig. 3. a Mean and standard deviation of relative tissue resistance $\mathrm{R}_{\text {rel }}^{\prime}$ in the CA1 field from five slices at fifteen intervals of half the electrode distance at a current frequency of $1 \mathrm{kHz}$. b Relative conductivities calculated from the mean values in a and smoothed, except at stratum pyramidale $(P)$ and the adjacent positions; A: alveus, Or: stratum oriens, Rad: stratum radiatum, Fi: hippocampal fissure

Because the intervals between the centres of the recording electrodes $(0.132 \mathrm{~mm})$ were more than twice the width of the pyramidal layer in CA1 $(0.04 \mathrm{~mm})$, we had to increase the spatial resolution in order to estimate the conductivity of this small layer. Therefore we used two different methods. With the first one we moved the electrode array by half the electrode interval $(0.066 \mathrm{~mm})$ and conducted a new series of measurements at several depths (Fig. 2b). Then for each slice the values $R_{\text {rel }}^{\text {of }}$ fifteen half electrode intervals were calculated from 


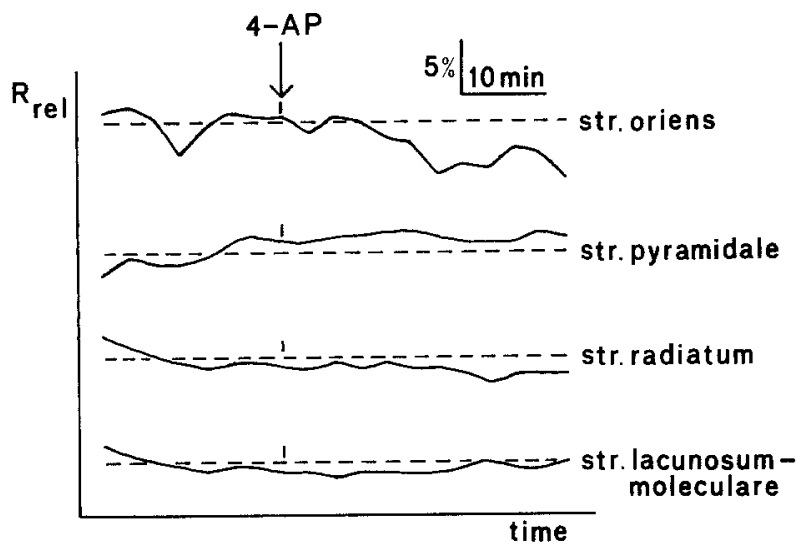

Fig. 4. Relative tissue resistance $\mathrm{R}_{\mathrm{rel}}$ in several layers of the $\mathrm{CA1}$ field before and after the application of 4-aminopyridine and the subsequent spontaneous generation of interictal bursts

the two series of seven mean values of $R_{\text {rel }}$, with str. pyramidale at electrode 3 and in-between electrodes 3 and 4 . For this calculation by subtraction we started in the middle part of str. radiatum, where the gradient of $R_{\text {rel }}$ was smallest. In this area we assumed that $R_{\text {rel }}^{\prime}$ in two half electrode intervals had the same value. From the values $\mathrm{R}_{\text {rel }}$ in 5 slices the mean and standard deviation were calculated for all fifteen $0.066 \mathrm{~mm}$ intervals (Fig. 3a). The lowest values $(0.88)$ were found in the middle part of str. radiatum. There was some increase towards the fissure (0.9-1.1) and the pyramidal layer. A peak value (1.5-1.6) was found in the pyramidal layer, and in str. oriens a somewhat higher value $(1.0-1.25)$ was found than in str. radiatum. In the alveus the results showed a large variation, probably due to the position of the first electrode, which was almost at the border of the slice with its tip not always surrounded by alvear tissue. In comparison with the normal width of the pyramidal layer in CA1 $(0.04 \mathrm{~mm})$ a spatial resolution of $0.066 \mathrm{~mm}$ is still not sufficient for a good estimation of $R_{r e l}$ in this layer. However, the pyramidal layer of some slices was almost as wide as the interval between two electrodes. We again recorded before and after moving the electrode array by half the electrode interval and calculated the results from the two recording series. In these experiments the peak value of $R_{\text {rel }}^{\prime}$ in str. pyramidale was 2.0-2.1.

The second way to increase the spatial resolution was to use an electrode array with smaller intervals $(0.08 \mathrm{~mm})$. After interpolation of the results we also got peak values of $\mathrm{R}_{\text {rel }}=2.0-2.2$ in str. pyramidale. In these experiments $R_{\text {rel }}$ in the middle part of str. radiatum was also normalized at a value of 0.88 .
For the calculation of the laminar profile of relative conductivity in CA1 we used the 15 average values from Fig. $3 \mathrm{a}$, except for $\mathrm{R}_{\text {rel }}$ in str. pyramidale for which we took the more reliable mean value 2.05 obtained from the latter experiments. We can expect that the conductivity changes gradually, except at the borders of str. pyramidale. Therefore the curve of $R_{\text {rel }}$ was smoothed by averaging over 3 successive values, except at str. pyramidale and adjacent positions. The inverse values - with a mean of 1.0 - are the relative conductivities $\left(\sigma_{\text {rel }}\right)$ shown in Fig. $3 b$. Starting from the alveus the profile increases almost linearly from 0.80 to 1.15 , only interrupted by a dip $(0.49)$ at str. pyramidale. Beyond the maximum in the middle of str. radiatum $\sigma_{\text {rel }}$ decreases to 0.96 at about the fissure. Because the current density in the slice cannot be established, we could only obtain relative values of $\sigma$, but these are sufficient for the purpose of CSD analysis.

\section{Laminar conductivity during epileptiform activity}

We were interested in the analysis of field potentials in the CA1 area of hippocampal slices during epileptiform activity, provoked by 4-aminopyridine (4-AP). Therefore conductivity measurements were carried out at $3 \mathrm{~min}$ intervals before and after application of 4-AP. 1-2 min after the application of 4-AP to the bathing medium spontaneous bursts started to occur regularly every $2-3 \mathrm{~s}$. With the application of current at a frequency of $1 \mathrm{kHz}$, we were able to avoid disturbance of the conductivity measurements by the spontaneous bursts, which had amplitudes of several milli-Volts and a frequency range up to approximately $800 \mathrm{~Hz}$. No significant changes in laminar conductivities, correlated with the application of 4-AP or with the onset of spontaneous bursts, were observed. Only slow fluctuations of $R_{\text {rel }}$ were observed, which did not exceed $\pm 2 \%$, except for str. oriens. In this layer $R_{\text {rel }}$ varied $\pm 5 \%$ (Fig. 4). Therefore it was concluded that laminar conductivities in the hippocampus do not change during epileptiform activity and that the values of relative conductivity obtained under normal conditions can also be used for CSD analysis of interictal bursts.

\section{Application to current density analysis}

The results of the laminar conductivity measurements in the hippocampal CA1 field were used for the inhomogeneous approximation of CSD analysis (see Appendix). This analysis was applied to field potentials, recorded with identical electrode arrays as 


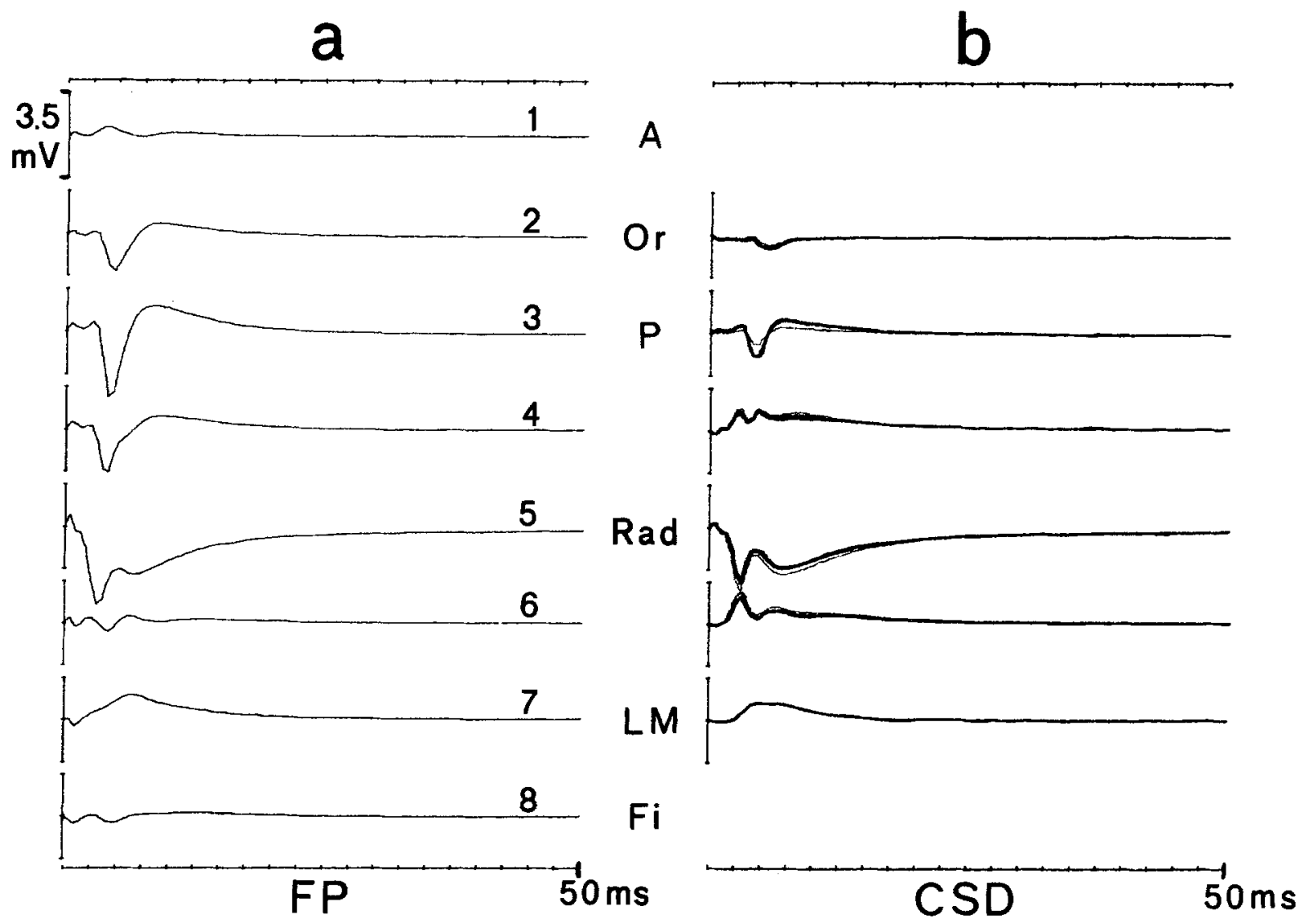

Fig. 5. a Simultaneous field potential recordings in the CA1 field of the hippocampal slice preparation with an array of eight electrodes at intervals of $0.13 \mathrm{~mm}$, perpendicular to the laminated structure (Fig. 1); averaged responses evoked by Schaffer collateral stimulation; positive upwards. b Current density calculated from the field potentials in a by the homogeneous (thick line) and the inhomogeneous (thin line) approximation; sinks downward; A: alveus, Or: stratum oriens, P: stratum pyramidale, Rad: stratum radiatum, LM: stratum lacunosum-moleculare, Fi: hippocampal fissure

used for the conductivity measurements and in the same position in the hippocampal slices. In Fig. 5a the response to Schaffer collateral stimulation, recorded simultaneously by the eight electrodes, is shown. At the fifth recording (str. radiatum) the negative field potential - due to excitatory postsynaptic currents - has its maximum, while at the third electrode (pyramidal layer) the subsequent population spike has its maximum. In Fig. 5b the current densities $I_{m}$, calculated for a homogeneous medium (fat line) and for an inhomogeneous medium (thin line) are shown. (See formulas [4] and [5] in the Appendix.) At the recording electrodes 2-7 we used the following values as the relative conductivity $\sigma\left(z_{0}\right)$ for the inhomogeneous approximation: $0.88 / 0.49$ / 1.05/1.14/1.14/1.01. The first derivatives $\left(\mathrm{d} \sigma\left(\mathrm{z}_{\mathrm{o}}\right) / \mathrm{dz}\right)$ at the electrodes $2-7$ were also derived from Fig. $3 b$ and are: $0.09 / 0.06 / 0.12 / 0.04 /-0.07 /-0.10$. Thus relatively small values of the conductivity gradient exist at the electrode positions. Larger values will be present at both sides of the pyramidal layer, between electrodes
2-3 and 3-4. In order to investigate the effect of these larger conductivity gradients, we had to calculate the current density at smaller intervals. Therefore eight simultaneous field potential values were interpolated by a third-order polynomial function (Rappelsberger et al. 1981). In this way three values were calculated between each pair of original field potential values, at intervals of $33 \mu \mathrm{m}$. In Fig. 6a the interpolated field potentials and their gradients are shown at the moment that the population spike at electrode 3 in Fig. 5a has its maximum value. The conductivities $\sigma\left(z_{o}\right)$ and their gradients at the 27 positions were estimated from Fig. 3b. Because the interpolated intervals $(33 \mu \mathrm{m})$ are more than half the width of the pyramidal layer, we assumed that the positions next to the electrode in the pyramidal layer were in str. oriens and str. radiatum and that the conductivities at these positions were almost the same as at the next positions in str. oriens and str. radiatum, respectively. Therefore, according to [5], the gradient of the conductivity only has a relatively large value at 

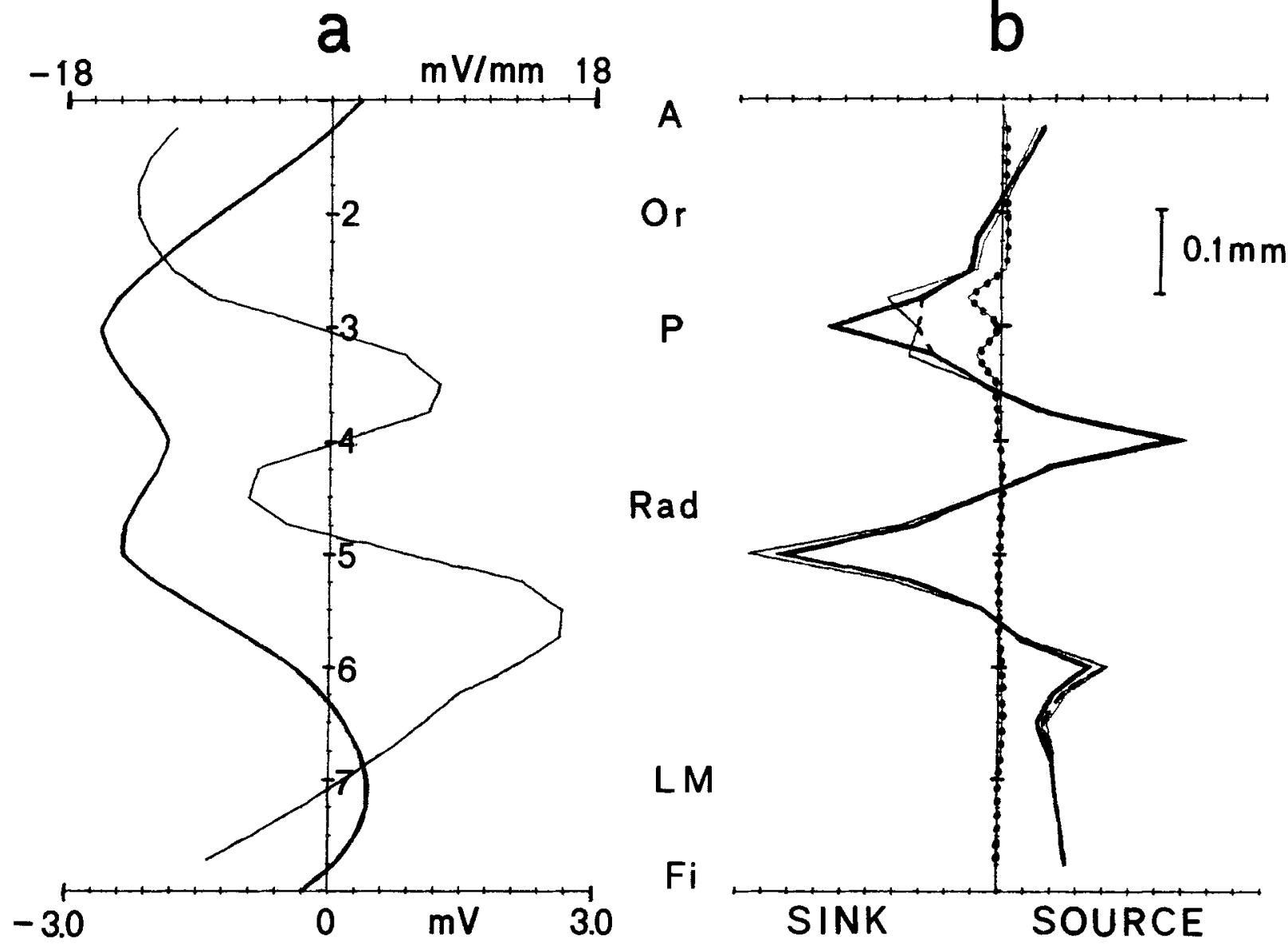

Fig. 6. a Interpolated field potential distribution (thick line, $-3 /+3 \mathrm{mV}$ ) and its gradient (thin line, $-18 /+18 \mathrm{mV} / \mathrm{mm}$ ) at $\mathrm{t}=4.5 \mathrm{~ms}$ of the experiment shown in Fig. 5a; positions of recording electrodes $(2-7)$ are indicated. b Current density calculated from the field potential distribution in a by the homogeneous (thick line) and the inhomogeneous (thin line) approximation. From the inhomogeneous approximation the part proportional to $\mathrm{d} \sigma\left(\mathrm{z}_{\mathrm{o}}\right) / \mathrm{dz}$ (dotted line) and the part proportional to $\sigma\left(\mathrm{z}_{\mathrm{o}}\right)$ (broken line) are also shown separately; A: alveus, Or: stratum oriens, P: stratum pyramidale, Rad: stratum radiatum, LM: stratum lacunosum-moleculare, Fi: hippocampal fissure

the positions next to str. pyramidale in str. oriens $(-0.22)$ and in str. radiatum $(0.25)$. At the other positions values range from -0.04 to 0.03 . In Fig. $6 \mathrm{~b}$ the current densities calculated by the homogeneous (thick line) and the inhomogeneous approximation (thin line), according to [4] and [5], are shown. The two parts of [5], proportional to the relative conductivity and to its gradient respectively, are also shown separately. The contribution of the last part (dotted line) is negligible, except at both sides of the pyramidal layer $(\mathrm{P})$, although the potential gradient at these positions is relatively small (Fig. 6a). The contribution of the first part of [5] (broken line) is almost identical to the homogeneous approximation, except at the pyramidal layer where the value of the sink is diminished by about $50 \%$. Hence, in comparison to the homogeneous approximation the inhomogeneous one gives almost identical results, except at and around the pyramidal layer. With the inhomogene- ous method the current density at str. pyramidale, found by the homogeneous method, is distributed over a wider area. At all positions $z_{0}$, except the two bordering the pyramidal layer, the (inhomogeneous) current densities have been changed almost proportionally to $\sigma\left(z_{0}\right)$ in comparison to the results of the homogeneous method. The current densities shown in Fig. 5b, calculated from eight field potentials at intervals of $0.132 \mathrm{~mm}$ for the whole response, are comparable with the results at the electrode position $2-7$ in Fig. 6b. Corresponding results were also obtained from other experiments.

\section{Discussion}

\section{Conductivity measurements}

A modified four-electrode method was used for the measurement of the relative conductivities of the 
layers in the hippocampal CA1 field. Instead of two, we had an array of eight recording electrodes between the current electrodes. In order to avoid current leakage through the recording electrodes high impedance preamplifiers were used. At $1 \mathrm{kHz}$ the input impedance calculated from the specifications $\left(10^{15} \Omega / / 0.6 \mathrm{pF}\right)$ was $280 \mathrm{M} \Omega$. At this frequency the impedance of the recording electrodes is $2-4 \mathrm{M} \Omega$ (Verloop and Holsheimer 1984), so that the amplitude of the recorded potentials was about $99 \%$ of the amplitudes in the nervous tissue at the electrode tips. In addition an isolated current source was used to avoid leakage via the reference electrode of the recording system, mounted at the bottom of the slice chamber. The current field from an isolated source is defined better than in a situation in which an unknown part of the current passes this reference electrode. If the current electrodes are just underneath the surface of the physiological salt solution without a slice in between, the current field in a small layer under the surface will be fairly homogeneous. But with a slice in this medium a different situation arises. The specific resistance of brain tissue is approx. $300 \Omega \mathrm{cm}$ (Lang et al. 1969), while a physiological salt solution or CSF has a value of approx. $54 \Omega \mathrm{cm}$ at $32^{\circ} \mathrm{C}$ (Geddes and Baker 1967). Thus the ratio between the mean specific conductivity of the slice and the specific conductivity of the surrounding medium is approx. $0.18: 1$, while in the slice laminar conductivities have a maximal ratio of approx. $2: 1$ (dendritic layers and pyramidal layer, respectively). This means that in the experimental situation we have a rather complex, inhomogeneous conducting medium. Therefore we were not able to calculate absolute, but only relative values of conductivity.

On top of a slice a small layer of bathing medium $(0.02-0.05 \mathrm{~mm})$ is present, with a specific resistance of approx. 0.18 times the average specific resistance of the slice. The effect of this shunt resistance will be that the differences in laminar resistance we measured are somewhat smaller than the real differences in $\mathbf{R}_{\mathrm{rel}}$. Because we do not know the exact thickness of this small layer, the shunt resistance is unknown and we cannot quantify the effect on our measurements. However, we have the same experimental situation when recording field potentials generated by the slice. Therefore it can be stated that the laminar conductivities calculated from the experiments are reliable for the purpose of CSD analysis of field potentials recorded under the same conditions.

The mean value of $R_{\text {rel }}$ measured in the alveus was low in comparison to the value expected in this layer of mainly nerve fibres and in a direction perpendicular to these fibres (Geddes and Baker 1967). In the experimental situation, however, the alveus - at the edge of the slice - is almost surrounded by a good conducting medium, which is a shunt for the alvear resistance. This may explain the relatively low value of $R_{\text {rel }}$ in the alveus. The large variation of this value among slices (Fig. 3a) may be due to differences in the thickness of the layer of bathing medium on top of the alveus, i.e. the value of the shunt resistance. That these variations are mainly confined to the edge of the slice can be seen in Fig. 4 . In this figure the fluctuations of $\mathrm{R}_{\mathrm{rel}}$ in str. oriens are larger than in the layers more to the centre of the slice. However, we do not have to use the (unreliable) value of relative conductivity in the alveus for the purpose of CSD-analysis.

Our measurements of laminar resistance at frequencies of $100 \mathrm{~Hz}$ and $1000 \mathrm{~Hz}$ gave exactly the same results. Because cellular membranes have a frequency-dependent conductivity, this result indicates that, at least up to $1000 \mathrm{~Hz}$, the proportion of current passing cellular membranes (and intracellular space) is negligible and all current in the slice will pass through the extracellular space (ES). Therefore the values of relative laminar conductivity obtained in this study can be used for the CSD analysis of all types of activity recorded in the hippocampus. Under the assumption that the specific extracellular conductivity and the tortuosity in the CA1 area are constant, the (laminar) conductivity will be proportional to the ES-fraction (Gardner-Medwin 1980). If we also assume that the width of ES between cellular elements is identical in all layers, conductivity will decrease with increasing diameter of the cellular elements. This relation explains the low conductivity of the pyramidal layer as compared to the dendritic layers. It may also provide an explanation for the gradual decrease of conductivity towards the alveus and the fissure (Fig. 3b). In both directions the axonal densities (of the pyramidal efferents and the perforant path fibres) increase, and an increasing number of axons between the smaller dendritic and presynaptic arborizations will decrease the mean ES.

Although the mean potential difference $\mathrm{dV}$ between the electrode pairs slightly decreased with increasing depth $(0.0-0.2 \mathrm{~mm})$, the ratio of $\mathrm{dV}$ from the electrode pairs did not change. Therefore it can be concluded that differences in physiological condition at several depths in the slice, due to differences in $\mathrm{pO}_{2}$ (Bingmann and Kolde 1982), do not affect the ratio of laminar conductivities. The decrease of the mean $\mathrm{dV}$ at increasing depth may be due to a slightly decreasing current density at deeper levels, or to a small increase of the ES fraction in all layers.

In the sensorimotor cortex of the cat Dietzel et al. (1980) observed a decrease of $30-50 \%$ of ES at sites of maximum rises in $[\mathrm{K}]_{\mathrm{o}}$ during and subsequent to 
ictal discharges. On the other hand they observed an increase of ES in other cortical layers. Since in the hippocampus the maximum increase of $[\mathrm{K}]_{0}$ during ictal and interictal activity is found in the pyramidal layer (Fisher et al. 1976), a decrease of conductivity in this layer and an increase in str. radiatum and str. oriens could be expected. However, in our experiments hippocampal laminar conductivities did not change after the onset of spontaneous interictal activity provoked by 4 -AP. Interictal activity consisted of a burst of 3-4 population spikes and occurred every 2-3 s. If we assume that the specific extracellular conductivity does not change, it can be concluded that significant changes in ES do not occur as a result of the interictal discharges in the hippocampal CA1 field. The potential differences monitored on an oscilloscope did not show transient changes in amplitude correlated with the spontaneous bursts either.

\section{CSD analysis}

It can be concluded from the results of this analysis that the effect of the inhomogeneous medium of the hippocampal CA1 field on the current density is proportional to the local conductivity, except at the borders of the pyramidal layer. The conductivity varies only slightly $(0.82-1.15)$, with an exception at this layer $(0.49)$. Conductivity gradients are small, except at the borders of the pyramidal layer. Therefore the only obvious difference between the current densities calculated by the homogeneous and the inhomogeneous approximation is found at and around the pyramidal layer. There is a decrease in current density here, while at the next positions in str. oriens and str. radiatum there is an increase. As in the example shown in Fig. 6b, this may result in two small peaks at both sides of str. pyramidale. However, in this example the sink at the pyramidal layer originates from simultaneous action potentials in the pyramidal somata (population spike). Therefore a single peak could be expected at the pyramidal layer instead of two peaks. Hence we have to conclude that the double peak is an artefact, due to the relatively large values of the conductivity gradient we calculated at the positions next to the pyramidal layer, together with the potential gradients at these positions. In fact we do not know exactly the conductivity gradients at the borders of the pyramidal layer and probably the values we used were too large. Another uncertainty is the exact position of the recording electrode in the pyramidal layer: in the middle or closer to one of its borders, e.g. symmetrical or asymmetrical between the large conductivity gradients. From the almost identical results of several experiments we can conclude that this electrodeposition in the pyramidal layer is not critical. Therefore the only obvious difference between the current densities is a flattened peak at the pyramidal layer with the inhomogeneous approximation, as compared to the homogeneous one. No sinks or sources appear or disappear. Therefore we may conclude that no significant difference exists between the homogeneous and the inhomogeneous approximation of the current density in the hippocampal CA1 field, except the large difference in conductivity between the pyramidal layer and the other layers.

\section{Appendix}

Current-source-density analysis

For the investigation of the spatial field resulting from synchronized neuronal activity (evoked potentials, epileptiform activity) in the hippocampal CA1 field, a one-dimensional CSDanalysis in a direction parallel to the axis of the pyramidal cells ( $\mathrm{Z}$ axis) can be used. CSD-analysis is based on the following equation (Nicholson and Freeman 1975):

$I_{m}\left(z_{o}, t\right)=-\frac{d \sigma\left(z_{0}\right)}{d z} \cdot \frac{d V_{e}\left(z_{0}, t\right)}{d z}-\sigma\left(z_{o}\right) \frac{d^{2} V_{e}\left(z_{o}, t\right)}{d z^{2}}$

in which $\sigma\left(z_{0}\right)$ is the tissue conductivity, $V_{e}\left(z_{o}, t\right)$ the field potential and $I_{m}\left(z_{0}, t\right)$ an approximation of the transmembrane current density at $z_{0}$.

If the medium is considered to be homogeneous, we get:

$I_{m}\left(z_{0}, t\right)=-\sigma \frac{d^{2} V_{e}\left(z_{0}, t\right)}{d z^{2}}$

If field potentials $\mathrm{V}_{\mathrm{e}}$ are recorded at discrete intervals $\mathrm{h}$, the following expression is used for a homogeneous medium:

$I_{m}\left(z_{0}, t\right)=-\sigma\left[V_{e}\left(z_{0}-h, t\right)-2 V_{e}\left(z_{0}, t\right)+V_{e}\left(z_{0}+h, t\right)\right] / h^{2}$

Since $\sigma$ and h are constant, they are only scale factors. With $\sigma=1$ and $\mathrm{h}=1$ we obtain:

$I_{m}\left(z_{0}, t\right)=-\left[V_{e}\left(z_{0}-h, t\right)-2 V_{e}\left(z_{0}, t\right)+V_{e}\left(z_{0}+h, t\right)\right]$

For an inhomogeneous medium we use:

$\mathrm{d} \sigma\left(\mathrm{z}_{\mathrm{o}}\right) / \mathrm{dz}=\left[\sigma\left(\mathrm{z}_{\mathrm{o}}+\mathrm{h} / 2\right)-\sigma\left(\mathrm{z}_{\mathrm{o}}-\mathrm{h} / 2\right)\right] / \mathrm{h}$

With $h=1$ we get the following expression:

$I_{m}\left(z_{0}, t\right)=-\left[\left(V_{e}\left(z_{0}-h, t\right)-2 V_{e}\left(z_{o}, t\right)+V_{e}\left(z_{o}+h, t\right)\right) \cdot \sigma\left(z_{0}\right)+\right.$ $\left.\left(\sigma\left(z_{\mathrm{o}}+\mathrm{h} / 2\right)-\sigma\left(\mathrm{z}_{\mathrm{o}}-\mathrm{h} / 2\right)\right) \cdot\left(\mathrm{V}_{\mathrm{e}}\left(\mathrm{z}_{\mathrm{o}}+\mathrm{h} / 2, \mathrm{t}\right)-\mathrm{V}_{\mathrm{e}}\left(\mathrm{z}_{\mathrm{o}}-\mathrm{h} / 2, \mathrm{t}\right)\right)\right]$

Acknowledgements. I would like to thank J.H. Koolstra and G.J. Heupink for their assistance with the experiments and A.J. Verloop for preparing the electrode arrays. I am also gratefull to Dr. F.H. Lopes da Silva for his useful suggestions, Ms. G.G.M. Steijlen for typing the manuscript and J. Baxter for correcting the English. 


\section{References}

Andersen P, Bliss TVP, Skrede KK (1971) Unit analysis of hippocampal population spikes. Exp Brain Res 13: 208-221

Bingmann D, Kolde $\mathrm{G}$ (1982) $\mathrm{PO}_{2}$-profiles in hippocampal slices of the guinea pig. Exp Brain Res 48: 89-96

Dietzel I, Heinemann U, Hofmeier C, Lux HD (1980) Transient changes in the size of the extracellular space in the sensorimotor cortex of cats in relation to stimulus-induced changes in potassium concentration. Exp Brain Res 40: 432-439

Fisher RS, Pedley TA, Moody WJ, Prince DA (1976) The role of extracellular potassium in hippocampal epilepsy. Arch Neurol 33: 76-83

Freeman JA, Stone J (1969) A technique for current density analysis of field potentials and its application to the frog cerebellum. In: Llinás $R$ (ed) Neurobiology of cerebellar evolution and development. Am Med Assoc, Chicago, pp 421-430

Gardner-Medwin AR (1980) Membrane transport and solute migration affecting the brain cell microenvironment. Neurosci Res Progr Bull 18: 208-226

Geddes LA, Baker LE (1967) The specific resistance of biological material - a compendium of data for the biomedical engineer and physiologist. Med Biol Eng 5: 271-293

Haberly LB, Shepherd GM (1973) Current-density analysis of summed evoked potentials in opossum prepyriform cortex. J Neurophysiol 36: 789-802

Hoeltzell PB, Dykes RW (1979) Conductivity in the somatosensory cortex of the cat - evidence for cortical anisotropy. Brain Res 177: 61-82
Kwan HC, Murphy JT (1974) A basis for extracellular current density analysis in cerebellar cortex. J Neurophysiol 37: 170-180

Lang J, Sances A, Larson SJ (1969) Determination of specific cerebral impedance and cerebral current density during the application of diffuse electrical currents. Med Biol Eng 7: $517-526$

Leung LS (1979) Orthodromic activation of hippocampal CA1 region of the rat. Brain Res 176: 49-63

Mitzdorf U (1985) Current source-density method and application in cat cerebral cortex: investigation of evoked potentials and EEG phenomena. Physiol Rev 65: 37-100

Mitzdorf U, Singer W (1978) Prominent excitatory pathways in the cat visual cortex (A17 and A18): a current source density analysis of electrically evoked potentials. Exp Brain Res 33: 371-394

Nicholson Ch, Freeman JA (1975) Theory of current source density analysis and determination of conductivity tensor for anuran cerebellum. J Neurophysiol 38: 356-368

Rappelsberger P, Pockberger H, Petsche H (1981) Current source density analysis: methods and application to simultaneously recorded field potentials of the rabbit's visual cortex. Pflügers Arch 389: 159-170

Schwartzkroin PA, Prince DA (1978) Cellular and field potential properties of epileptogenic hippocampal slices. Brain Res 147: $117-130$

Verloop AJ, Holsheimer J (1984) A simple method for the construction of electrode arrays. J Neurosci Methods 11: 173-178

Received August 28, 1986 / Accepted February 13, 1987 